\title{
The mid-infrared instrument for the James Webb Space Telescope: performance and operation of the Low-Resolution Spectrometer
}

Sarah Kendrew, Silvia Scheithauer, Patrice Bouchet, Jerome Amiaux, Ruymán Azzollini, et al.

Sarah Kendrew, Silvia Scheithauer, Patrice Bouchet, Jerome Amiaux, Ruymán Azzollini, Jeroen Bouwman, Christine Chen, Didier Dubreuil, Sebastian Fischer, Ori D Fox, Alistair Glasse, Karl Gordon, Tom Greene, Dean C. Hines, Pierre-Olivier Lagage, Fred Lahuis, Samuel Ronayette, David Wright, Gillian S. Wright, "The mid-infrared instrument for the James Webb Space Telescope: performance and operation of the Low-Resolution Spectrometer," Proc. SPIE 9904, Space Telescopes and Instrumentation 2016: Optical, Infrared, and Millimeter Wave, 990443 (4 August 2016); doi: 10.1117/12.2232887

Event: SPIE Astronomical Telescopes + Instrumentation, 2016, Edinburgh, United Kingdom 


\title{
The Mid-Infrared Instrument for the James Webb Space Telescope: performance and operation of the Low-Resolution Spectrometer
}

\author{
Sarah Kendrew ${ }^{\mathrm{a}, \mathrm{b}, \mathrm{c}}$, Silvia Scheithauer ${ }^{\mathrm{c}}$, Patrice Bouchet ${ }^{\mathrm{d}}$, Jerome Amiaux ${ }^{\mathrm{d}}$, Ruymán \\ Azzollini ${ }^{\mathrm{e}, \mathrm{f}}$, Jeroen Bouwman ${ }^{\mathrm{b}}$, Christine Chen ${ }^{\mathrm{g}}$, Didier Dubreuil ${ }^{\mathrm{d}}$, Sebastian Fischer ${ }^{\mathrm{h}, \mathrm{i}}$, Ori D. \\ Fox $^{\mathrm{g}}$, Alistair Glasse ${ }^{\mathrm{j}}$, Karl Gordon ${ }^{\mathrm{g}}$, Tom Greene ${ }^{\mathrm{k}}$, Dean C. Hines ${ }^{\mathrm{g}}$, Pierre-Olivier Lagage ${ }^{\mathrm{d}}$, \\ Fred Lahuis ${ }^{1, m}$, Samuel Ronayette ${ }^{\mathrm{d}}$, David Wright ${ }^{\mathrm{n}}$, and Gillian S. Wright ${ }^{\mathrm{j}}$ \\ aEuropean Space Agency, Space Telescope Science Institute, Baltimore, USA \\ ${ }^{b}$ University of Oxford, Oxford, UK \\ 'Max-Planck-Institut für Astronomie, Heidelberg, Germany \\ d Laboratoire AIM Paris-Saclay, Gif-sur-Yvette, France \\ eUniversity College London, London, UK \\ ${ }^{\mathrm{f}}$ Dublin Institute for Advanced Studies, Dublin, Ireland

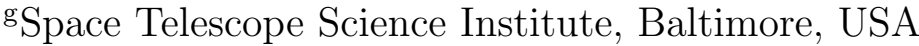 \\ ${ }^{\mathrm{h}}$ Deutsches Zentrum für Luft- und Raumfahrt (DLR), Bonn, Germany \\ ${ }^{i}$ I. Physikalisches Institut, Universität zu Köln, Köln, Germany \\ jUK Astronomy Technology Centre, Edinburgh, UK \\ ${ }^{\mathrm{k}}$ Ames Research Center, Moffett Field, USA \\ ISRON Groningen, Groningen, The Netherlands \\ ${ }^{\mathrm{m}}$ University of Leiden, Leiden, The Netherlands \\ nStinger Ghaffarian Technologies, Inc., Greenbelt, USA
}

\begin{abstract}
We describe here the performance and operational concept for the Low Resolution Spectrometer (LRS) of the mid-infrared instrument (MIRI) for the James Webb Space Telescope. The LRS will provide R 100 slit and slitless spectroscopy from 5 to 12 micron, and its design is optimised for observations of compact sources, such as exoplanet host stars. We provide here an overview of the design of the LRS, and its performance as measured during extensive test campaigns, examining in particular the delivered image quality, dispersion, and resolving power, as well as spectrophotometric performance. The instrument also includes a slitless spectroscopy mode, which is optimally suited for transit spectroscopy of exoplanet atmospheres. We provide an overview of the operational procedures and the differences ahead of the JWST launch in 2018.
\end{abstract}

Keywords:

\section{OVERVIEW AND DESIGN DESCRIPTION}

The Mid-Infrared Instrument (MIRI) is one of four science instruments for the James Webb Space Telescope, set to be launched in 2018. As the only mid-infrared instrument on board JWST, MIRI provides unprecedented sensitivity, sub-arcsecond angular resolution, and continuous wavelength coverage from 5 to $28 \mu \mathrm{m}$, free from atmospheric interference $^{1,2 *}$. It offers a suite of versatile observing capabilities, including imaging, low- resolution slit and slitless spectroscopy, medium-resolution integral field spectroscopy, and coronagraphy. The instrument will address a number of major science topics:

\footnotetext{
Send correspondence to S. Kendrew, ESA, sarah.kendrew@esa.int

*http://ircamera.as.arizona.edu/MIRI/encyclopedia.htm
} 


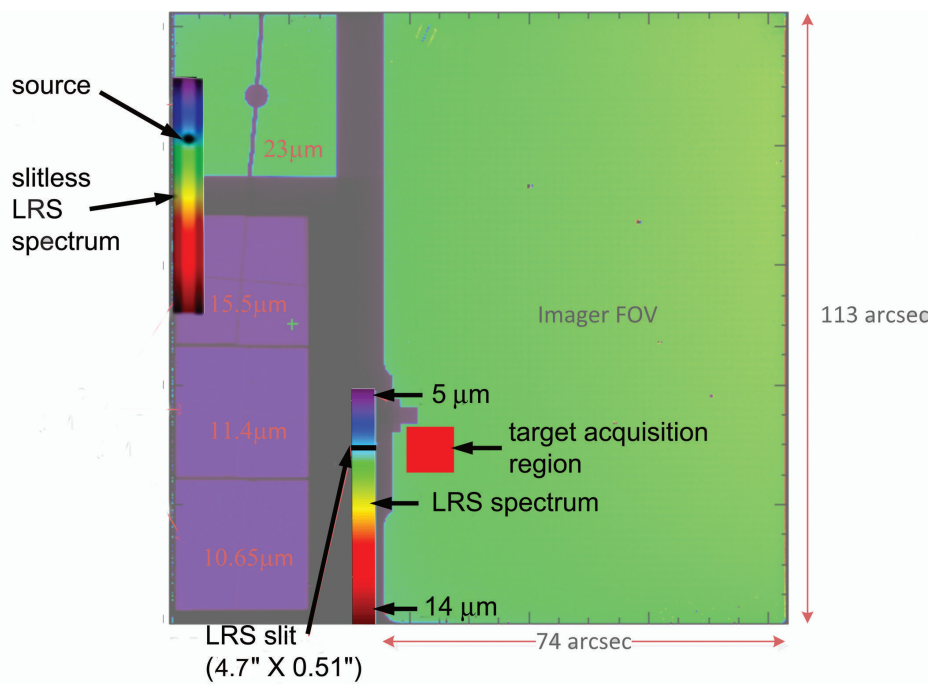

Figure 1. Schematic view of the MIRIM focal plane highlighting the locations of the (fixed) $5.5^{\prime \prime}$ LRS slit, and the LRS $7^{\prime \prime}$ wide slitless readout window. The red box shows the approximate location of the $10^{\prime \prime} \times 10^{\prime \prime}$ target acquisition region for slit spectroscopy. Not labelled in the figure is the 'SUB64' imaging subarray $(64 \times 64$ pixels in size $)$, which is located within the slitless subarray region; this subarray will be used for target acquisition for slitless observations.

- Exoplanet imaging and transit spectroscopy

- The early stages of star and planet formation

- Galaxy assembly over cosmic time

- The first galaxies.

The LRS forms part of the MIRI Imager module (MIRIM ${ }^{3}$ ), and will deliver R 100 spectroscopy from 5 to $12 \mu \mathrm{m}$, optimised for spectroscopy of compact sources such as high-redshift galaxies or exoplanet host stars. The spectrometer can be operated in slit or slitless mode, with the spectrum dispersed by a double prism assembly (DPA) mounted in the imager filter wheel. The entrance slit is $4.7^{\prime \prime}$ long and $0.51^{\prime \prime}$ wide when projected onto the sky, corresponding to 43 pixels and 4.6 pixels respectively on the array. A diagram of the focal plane layout of MIRIM is shown in Figure 1, with key locations for the LRS indicated.

The as-designed dispersion and spectral resolving power are shown in Figure 2. To mitigate the effects of the spectrum fold-over short of $4.5 \mu \mathrm{m}$, a filter is mounted onto the slit mask; this does however reduce the transmission of the LRS in enslitted compared with slitless mode. We discuss this and further differences between the two modes of operation for LRS in Section 3.

Whilst observing with LRS there is no way of blocking the imager portion of the focal plane. Any point sources in this part of the field will therefore be dispersed as slitless spectra. This raises the possibility of light scattering into the LRS detector region, and saturation, causing detrimental effects on subsequent exposures. The location of the spectrum of a source through the slit was chosen such that there would be no overlap with the spectrum of any point sources in the Lyot coronagraph field-of-view.

A detailed description of the design, build and operations of the LRS can be found in Kendrew et al (2015). ${ }^{4}$ Operations and calibration of the full instrument, including LRS, are described by Gordon et al (2015). ${ }^{5}$

\section{PERFORMANCE}

The performance of the LRS has been tested over the course of several test campaigns. Initial testing was performed on the MIRIM module alone after assembly of the flight hardware in 2009-2010 at CEA, Paris. This allowed a first characterization of the imager, coronagraph and LRS performance, however these results were not 

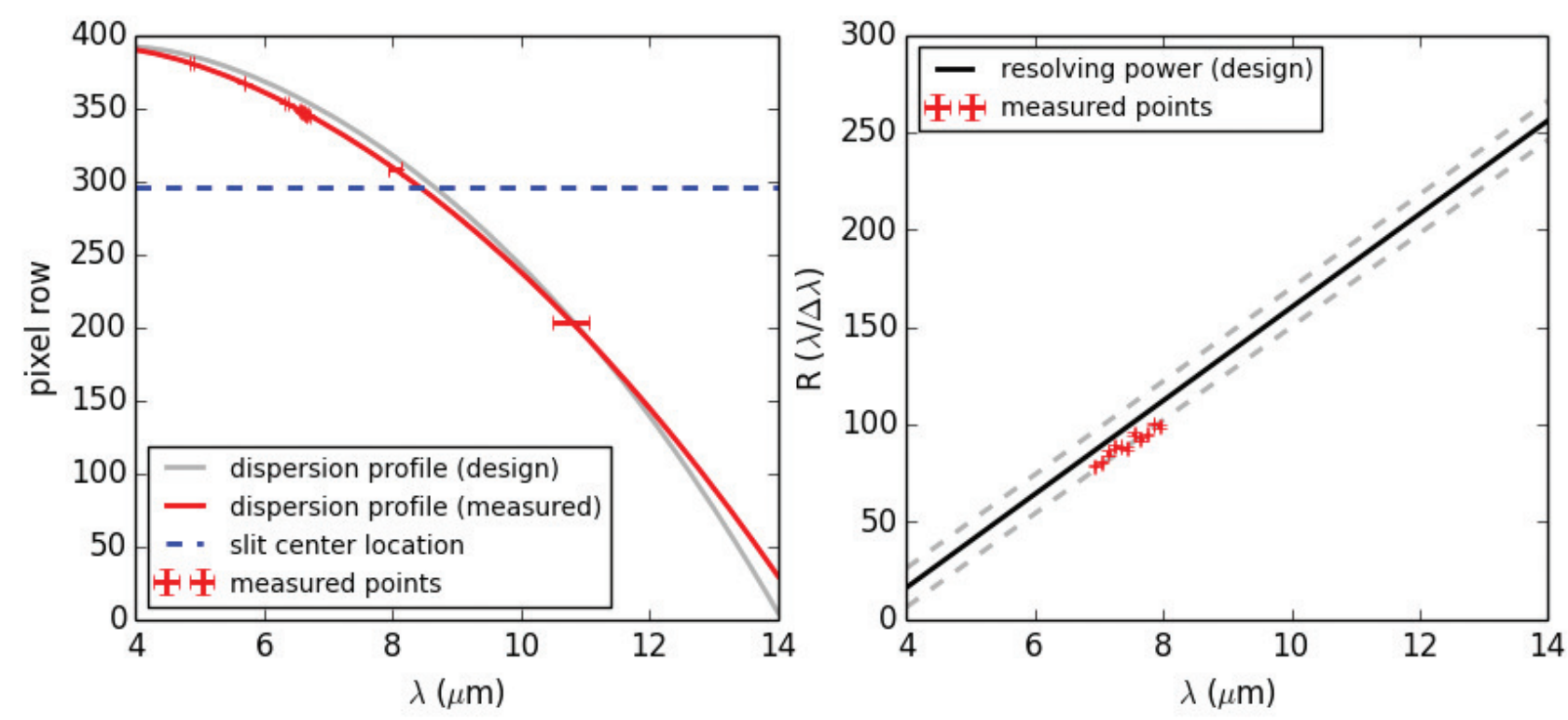

Figure 2. Left: As-designed and measured spectral dispersion profile of the LRS between 4 and $14 \mu \mathrm{m}$. The position of the slit center is shown with the dashed line. Right: As-designed and measured spectral resolving power of LRS. The resolving power varies linearly with wavelength, with $\mathrm{R}=100$ at $\lambda=7.5 \mu \mathrm{m}$. The dashed lines show the upper and lower bounds of the design specification.

fully representative of the final instrument system. In addition, at this stage the MIRI Imager did not include the flight detectors; radiometric measurements were thus not made. This testing phase did provide the opportunity for performing valuable optical measurements and calibration tests.

Following assembly of the flight hardware, MIRI was subjected to several extensive test campaigns examining the optical and radiometric performance of all observing modes, at the Rutherford Appleton Laboratory in 2011. We summarize below the key findings of these tests for the LRS.

Point Spread Function (PSF). The LRS aims to provide near-diffraction-limited image quality over 5-10 $\mu \mathrm{m}$. Testing combined with optical modeling to account for ground test equipment artefacts revealed a spatial PSF of $2.6 \pm 0.1 \mathrm{px}$ at $7.7 \mu \mathrm{m}$; this is within $10-15 \%$ of the diffraction limit at this wavelength. Point source exposures were combined to create a PSF model to be used for spectral extraction.

Spectral distortion. The spatial distortion of the spectrum on the detector was measured and found to be very small. There is no evidence for variation in the distortion profile with the position of the source along the length of the slit within the uncertainty on the available measurements. The distortion is compensated as part of the calibration and extraction of the spectrum in the pipeline.

Wavelength calibration and spectral resolving power. Using ground testing equipment the LRS spectral resolving power and dispersion were measured during test campaigns from 2009 to 2011; these were found to be consistent with the as-designed values (see Figure 2). The accuracy of the wavelength calibration depends critically on the precise knowledge of the source on the detector. With the excellent pointing accuracy of JWST in flight, an accuracy of 1-2 $\mathrm{nm}$ is expected to be achieved in LRS wavelength calibration. A reference table was derived to perform the wavelength calibration in the data pipeline; this will be updated following the commissioning period, during which the wavelength calibration will be re-measured using astrophysical targets.

Flat fields. LRS flat fields will be produced using a combination of exposures with an on-board calibration source and sky flats. Initial flat fields were produced during the ground test campaigns using the calibration source, however sky flats are required for the accurate calibration of low spatial frequency variations. 

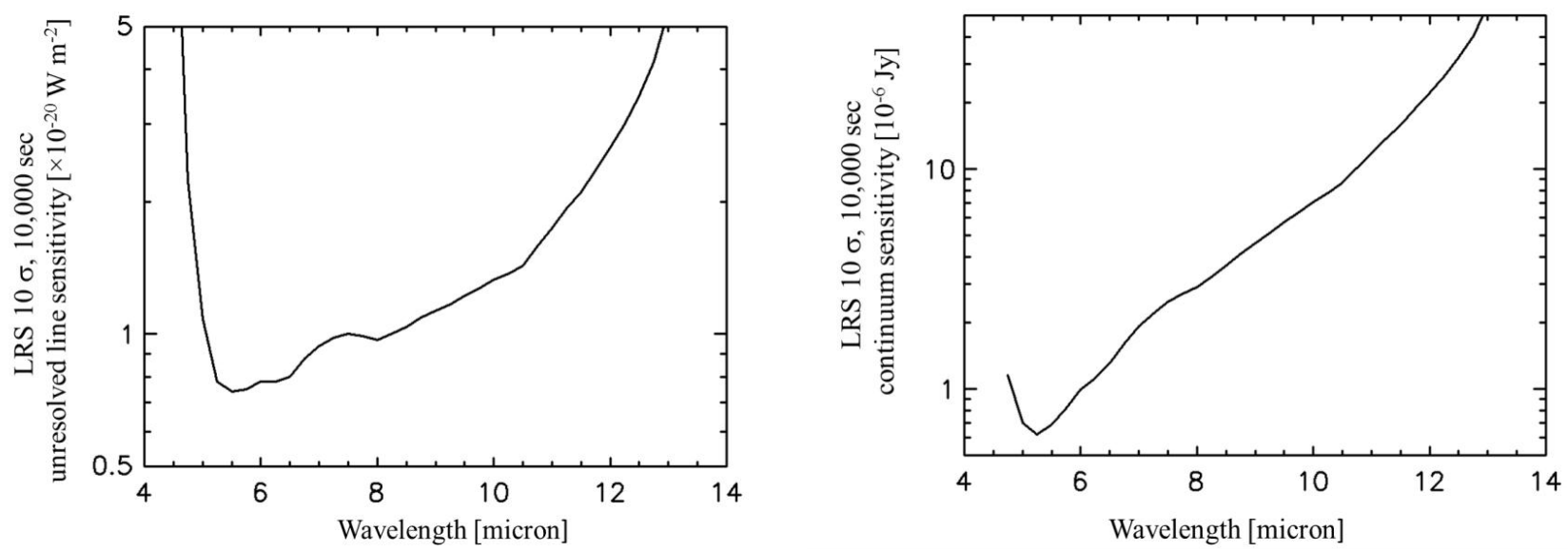

Figure 3. LRS sensitivity plots for point source observations to $10-\sigma$ in $10^{4}$ seconds. Left: sensitivity to an unresolved line (units $10^{-20} \mathrm{~W} \mathrm{~m}^{-2}$ ). Right: continuum sensitivity, in $\mu \mathrm{Jy}$.

Sensitivity. A comprehensive sensitivity model for the LRS (as well as imager and MRS) is described by Glasse et al $(2010,2015) .{ }^{6,7}$ The model incorporates the knowledge gained from the successive ground test phases with MIRI. It includes, amongst others, the effects of the observatory thermal background, detector sampling, noise and quantum efficiency, and the possible effects of cosmic rays. The current model applies to LRS in slit mode; for slitless mode we expect a lower sensitivity. We note that corrections were made to the model of Glasse et al (2015) ${ }^{7}$ after publication; the corrected versions of the estimated LRS continuum and spectral line sensitivities are shown in Figure 3.

Bright source limit. Given the LRS' suitability for observations of exoplanet host stars, many of which are very bright, the bright source limit is relevant. This was calculated by Glasse et al $(2015)^{7}$ for the slit mode of LRS, and shown in Figure 4. For slitless LRS observations, a subarray was defined on the detector (see Figure 1) that can be read out in $0.16 \mathrm{~s}$, as opposed to $\sim 3 \mathrm{~s}$ for the full array (in 'FAST' readout mode ${ }^{8,9}$ ). This provides a factor 17 improvement in the bright source limit and allows for the observation of most of the currently known exoplanet host stars without the risk of saturation. ${ }^{4,7}$

Fringing. Spectral fringes are a common characteristic of mid-infrared spectrometers, ${ }^{10,11}$ typically resulting from optical interference in the detector substrate. Within MIRI, fringes are observed originating in the detector surface and filters in the MIRI optical path. In the LRS the fringes are unresolved and blend in with the spectral response function; for the MRS however they are resolved, we therefore used the MRS fringe characteristics to assess the impact of the fringes on LRS spectra. Modeling shows that under nominal conditions the effects of fringes will be accurately calibrated. However for extreme $\mathrm{S} / \mathrm{N}$ observations (e.g. bright exoplanet host stars), pointing jitter or drifts can become a significant noise component if not accounted for. The development of a modeling framework complemented with targeted in-orbit calibration observations will be important.

\section{OPERATIONS}

The operational concept and data reduction strategy for JWST-MIRI is described in detail by Gordon et al (2015). ${ }^{5}$ Observations with JWST are described by an observing template (OT), each of which provides an interface for astronomers to specify the sequence of steps requested for their programs. The knowledge of instrument experts is captured in these OTs; the principles that drive the OT design include redundant observations (e.g., multiple dithers), minimizing the use of the instrument mechanisms (e.g., filter and grating wheels), and creating the minimal set of options that accomplishes all expected MIRI science while ensuring robust data reduction and a homogeneous and high-quality archive. 


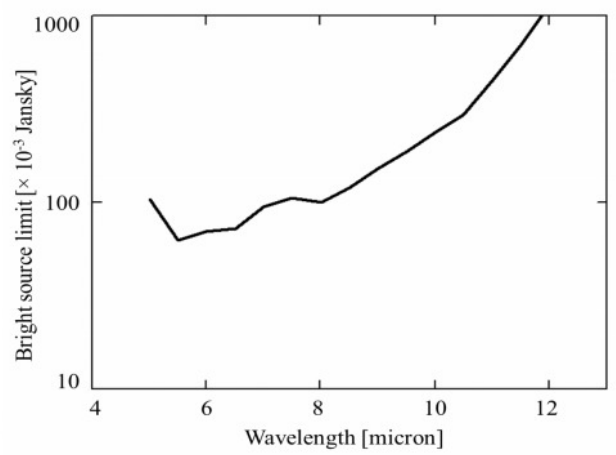

Figure 4. Bright source limits (assuming a worst case thermal background) for the MIRI LRS. The minimum value is $63 \mathrm{mJy}$ at $5.5 \mu \mathrm{m}$.

Low-resolution spectroscopy represents one of 4 OTs for MIRI (the others being imaging, coronagraphy and medium-resolution spectroscopy). For LRS the main components of the OT are target acquisition, the dither strategy, and slit vs. slitless operations.

For target acquisition (TA) into the slit, the LRS will make use of a small target acquisition region in the imager portion of the detector located $<20^{\prime \prime}$ from the slit centre location; within the maximum distance that can be covered by a small angle maneuver of the telescope. During TA, the source is placed in this region, imaged with a suitable filter, and its exact position then determined through centroiding. The TA region for slit spectroscopy is shown in Figure 1. For slitless spectroscopy, where targets are typically bright, an imager subarray ('SUB64'9) will be used for TA purposes.

Three dither strategies are offered for LRS observations: no dither (particularly suitable for time series observations), a two-point dither, or extended source/mapping observations. For point sources, the two-point dither places the source at the $1 / 3 \mathrm{rd}$ and $2 / 3 \mathrm{rd}$ along-slit locations, providing enough spatial coverage for background subtraction. For extended sources, the telescope will move entirely off the source for background measurements.

The main scientific driver for the slitless LRS mode is high precision spectrophotometry of bright nearby stars with transiting planets to obtain spectra of exoplanet atmospheres. When performed using slit spectroscopy, where the slit is of comparable width to the size of the PSF, throughput variations from pointing uncertainty and drifts can be the dominant source of systematic noise. ${ }^{12}$ Performing spectroscopy without the presence of a slit can avoid this issue. Second, the required S/N ratios for exoplanet transit spectroscopy can only be achieved for relatively nearby, bright transiting systems. Many of these targets are too bright to be observed with LRS slit spectroscopy, for which the minimum frame read time is $\sim 3 \mathrm{~s}$. To this end a subarray was defined for slitless spectroscopy, with a shortest read time of $\sim 0.16 \mathrm{~s} ;{ }^{8}$ this decreases the bright limit by 2 magnitudes (to $\mathrm{K} \sim 5.6$ ), allowing observations of many more exoplanet host stars.

In slitless mode, the light shortward of $4.5 \mu \mathrm{m}$ is not blocked by the filter mounted on the slit mask; the spectrum therefore displays the fold-over seen in the dipsersion profile (Figure 2). The lack of filter does however improve the overall transmission, particularly at longer wavelengths: at $12 \mu \mathrm{m}$, the transmission in slitless mode is approximately $60 \%$ higher than through the slit. The photon conversion efficiency (PCE) - the ratio between the electrons measured at the detector relative to the number of photons at the MIRI imager entrance focal plane (i.e. telescope optics are not included) - is also higher for slitless mode due to the absence of a slit. Comparison of the PCE for slit and slitless mode is shown in Figure 5.

\section{SUMMARY}

The MIRI Low Resolution Spectrometer provides near-diffraction-limited spectroscopy at $\mathrm{R} \sim 100$ from 5 to $12 \mu \mathrm{m}$. The module can be operated in slit or slitless mode, with the latter particularly suitable for time series observations such as exoplanet transits. In this paper we have described key outcomes of the test campaigns to 


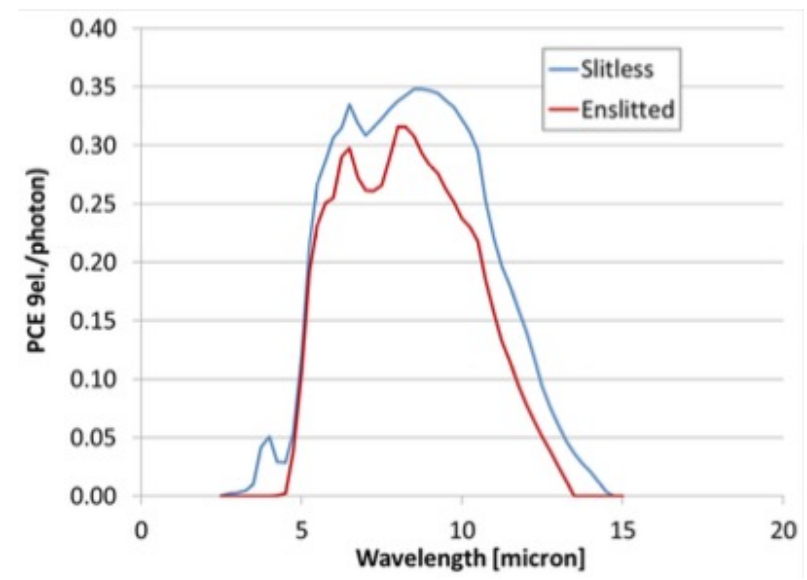

Figure 5. Photon conversion efficiencies (PCE) from LRS measured point source fluxes during flight testing. The red curve represents slit spectroscopy, the blue curve slitless. The feature around $8 \mu \mathrm{m}$ is an artefact, not physically representative of the instrument.

characterize the performance of the LRS mode, and given an overview of the operational concept. This is key to understanding the instrument's capabilities for low-resolution spectroscopy and the operational differences between the slit and slitless modes when planning future observations.

\section{REFERENCES}

[1] Rieke, G. H., Wright, G. S., Böker, T., Bouwman, J., Colina, L., Glasse, A., Gordon, K. D., Greene, T. P., Güdel, M., Henning, T., Justtanont, K., Lagage, P.-O., Meixner, M. E., Nørgaard-Nielsen, H.-U., Ray, T. P., Ressler, M. E., van Dishoeck, E. F., and Waelkens, C., "The Mid-Infrared Instrument for the James Webb Space Telescope, I: Introduction," PASP 127, 584-594 (July 2015).

[2] Wright, G. S., Wright, D., Goodson, G. B., Rieke, G. H., Aitink-Kroes, G., Amiaux, J., Aricha-Yanguas, A., Azzollini, R., Banks, K., Barrado-Navascues, D., Belenguer-Davila, T., Bloemmart, J. A. D. L., Bouchet, P., Brandl, B. R., Colina, L., Detre, Ö., Diaz-Catala, E., Eccleston, P., Friedman, S. D., García-Marín, M., Güdel, M., Glasse, A., Glauser, A. M., Greene, T. P., Groezinger, U., Grundy, T., Hastings, P., Henning, T., Hofferbert, R., Hunter, F., Jessen, N. C., Justtanont, K., Karnik, A. R., Khorrami, M. A., Krause, O., Labiano, A., Lagage, P.-O., Langer, U., Lemke, D., Lim, T., Lorenzo-Alvarez, J., Mazy, E., McGowan, N., Meixner, M. E., Morris, N., Morrison, J. E., Müller, F., rgaard-Nielson, H.-U. N., Olofsson, G., O'Sullivan, B., Pel, J.-W., Penanen, K., Petach, M. B., Pye, J. P., Ray, T. P., Renotte, E., Renouf, I., Ressler, M. E., Samara-Ratna, P., Scheithauer, S., Schneider, A., Shaughnessy, B., Stevenson, T., Sukhatme, K., Swinyard, B., Sykes, J., Thatcher, J., Tikkanen, T., van Dishoeck, E. F., Waelkens, C., Walker, H., Wells, M., and Zhender, A., "The Mid-Infrared Instrument for the James Webb Space Telescope, II: Design and Build," PASP 127, 595-611 (July 2015).

[3] Bouchet, P., García-Marín, M., Lagage, P.-O., Amiaux, J., Auguéres, J.-L., Bauwens, E., Blommaert, J. A. D. L., Chen, C. H., Detre, Ö. H., Dicken, D., Dubreuil, D., Galdemard, P., Gastaud, R., Glasse, A., Gordon, K. D., Gougnaud, F., Guillard, P., Justtanont, K., Krause, O., Leboeuf, D., Longval, Y., Martin, L., Mazy, E., Moreau, V., Olofsson, G., Ray, T. P., Rees, J.-M., Renotte, E., Ressler, M. E., Ronayette, S., Salasca, S., Scheithauer, S., Sykes, J., Thelen, M. P., Wells, M., Wright, D., and Wright, G. S., "The Mid-Infrared Instrument for the James Webb Space Telescope, III: MIRIM, The MIRI Imager," PASP 127, 612-622 (July 2015).

[4] Kendrew, S., Scheithauer, S., Bouchet, P., Amiaux, J., Azzollini, R., Bouwman, J., Chen, C. H., Dubreuil, D., Fischer, S., Glasse, A., Greene, T. P., Lagage, P.-O., Lahuis, F., Ronayette, S., Wright, D., and Wright, G. S., "The Mid-Infrared Instrument for the James Webb Space Telescope, IV: The Low-Resolution Spectrometer," PASP 127, 623-632 (July 2015).

[5] Gordon, K. D., Chen, C. H., Anderson, R. E., Azzollini, R., Bergeron, L., Bouchet, P., Bouwman, J., Cracraft, M., Fischer, S., Friedman, S. D., García-Marín, M., Glasse, A., Glauser, A. M., Goodson, G. B., 
Greene, T. P., Hines, D. C., Khorrami, M. A., Lahuis, F., Lajoie, C.-P., Meixner, M. E., Morrison, J. E., O'Sullivan, B., Pontoppidan, K. M., Regan, M. W., Ressler, M. E., Rieke, G. H., Scheithauer, S., Walker, H., and Wright, G. S., "The Mid-Infrared Instrument for the James Webb Space Telescope, X: Operations and Data Reduction," PASP 127, 696-711 (July 2015).

[6] Glasse, A. C. H., Bauwens, E., Bouwman, J., Detre, Ö., Fischer, S., Garcia-Marin, M., Justannont, K., Labiano, A., Nakos, T., Ressler, M., Rieke, G., Scheithauer, S., Wells, M., and Wright, G. S., "The throughput and sensitivity of the JWST mid-infrared instrument," in [Space Telescopes and Instrumentation 2010: Optical, Infrared, and Millimeter Wave], Proc. SPIE 7731, 77310K (July 2010).

[7] Glasse, A., Rieke, G. H., Bauwens, E., García-Marín, M., Ressler, M. E., Rost, S., Tikkanen, T. V., Vandenbussche, B., and Wright, G. S., "The Mid-Infrared Instrument for the James Webb Space Telescope, IX: Predicted Sensitivity," PASP 127, 686-695 (July 2015).

[8] Ressler, M. E., Sukhatme, K. G., Franklin, B. R., Mahoney, J. C., Thelen, M. P., Bouchet, P., Colbert, J. W., Cracraft, M., Dicken, D., Gastaud, R., Goodson, G. B., Eccleston, P., Moreau, V., Rieke, G. H., and Schneider, A., "The Mid-Infrared Instrument for the James Webb Space Telescope, VIII: The MIRI Focal Plane System," PASP 127, 675-685 (July 2015).

[9] Bright, S., Ressler, M. E., Alberts, S., Noriega-Crespo, A., Morrison, J. E., García-Marín, M., Fox, O., Rieke, G. H., Glasse, A. C., Wright, G. S., Hines, D. C., Bouchet, P., and Dicken, D., "MIRI/JWST Detector Characterization," in [Space Telescopes and Instrumentation 2016: Optical, Infrared and Millimeter wave], Proc. SPIE (2016).

[10] Lahuis, F. and Boogert, A., "How to Get Rid of Fringes in SIRTF/IRS Data," in [SFChem 2002: Chemistry as a Diagnostic of Star Formation], Curry, C. L. and Fich, M., eds., 335 (2003).

[11] Kester, D. J. M., Beintema, D. A., and Lutz, D., "SWS Fringes and Models," in [The Calibration Legacy of the ISO Mission], Metcalfe, L., Salama, A., Peschke, S. B., and Kessler, M. F., eds., ESA Special Publication 481, 375 (2003).

[12] Swain, M. R., Bouwman, J., Akeson, R. L., Lawler, S., and Beichman, C. A., "The Mid-Infrared Spectrum of the Transiting Exoplanet HD 209458b," ApJ 674, 482-497 (Feb. 2008). 